\title{
Application and calibration of a simple position detector for a dust accelerator
}

\author{
Katharina A. Otto ${ }^{\mathrm{a}, *}$, Ralf Srama ${ }^{\mathrm{a}, \mathrm{b}, * *}$, Siegfried Auer ${ }^{\mathrm{c}}$, Sebastian Bugiel ${ }^{\mathrm{a}, \mathrm{b}}$, \\ Eberhard Grün $^{\mathrm{a}, \mathrm{d}}$, Sascha Kempf ${ }^{\mathrm{d}}$, Jianfeng Xie ${ }^{\mathrm{d}}$ \\ a Max Planck Institut für Kernphysik, Saupfercheckweg 1, 69117 Heidelberg, Germany \\ ${ }^{\mathrm{b}}$ Institut für Raumfahrtsysteme, Universität Stuttgart, Pfaffenwaldring 29, 70569 Stuttgart, Germany \\ c AEM Associates, P.O. Box 421, Basye, VA 22810, USA \\ ${ }^{\mathrm{d}}$ Laboratory for Atmospheric and Space Physics, University of Colorado at Boulder, 1234 Innovation Drive, Boulder, CO 80303, USA
}

\section{A R T I C L E I N F O}

\section{Article history:}

Received 25 January 2013

Received in revised form

16 July 2013

Accepted 21 July 2013

Available online 31 July 2013

Keywords:

Position detector

Dust accelerator

Beam monitoring

Calibration

Electrical induction

Detector simulation

\begin{abstract}
A B S T R A C T
A newly developed position sensitive detector was implemented in the beam line of the Heidelberg dust accelerator. By charge induction, the detector enables the position of a dust particle to be determined without affecting its motion. The detector consists of four pairs of parallel plates, connected to a single common charge amplifier. The charge induced on the plates varies as a function of the dust particle trajectory, producing simple, easily interpreted signals. Using a segmented target installed in the beam line for a second independent measure of the trajectory, the position detector has been calibrated, allowing the detector signal to be mapped to a dust particle position. The resulting calibration curve indicates that the detector's position accuracy is approximately $0.14 \mathrm{~mm}$, based on an average SNR of 700 for dust particles passing through the centre of the detector. The minimum dust charge for reliable detection was found to be about $1.1 \mathrm{fC}$. A detector simulation was used to produce a calibration curve that confirms the experimental results.
\end{abstract}

(c) 2013 Elsevier B.V. All rights reserved.

\section{Introduction}

In the past five decades, various dust detection instruments on spacecrafts have been used successfully to investigate in situ the properties of cosmic dust in the Solar System [1-4]. Dust detectors are able to provide information of individual dust grains. This can be physical properties such as grain mass and charge determined by electrical induction. The chemical composition can be ascertained by dust impinging onto a target and evaluating the resulting impact ionisation plasma. Moreover, the orbital and dynamical properties can be measured using positional variation of induced charge by a dust grain. Modern dust instruments combine all these detectors [5].

Much effort has been put in the development of these instruments, however a major task before launching a new dust instrument is calibrating and testing it. For this reason an electrostatic dust accelerator was developed to accelerate charged micrometre-sized dust particles to hypervelocities, similar to those found in space [6,7]. The accelerator assembly is illustrated in Fig. 1. It allows dust

\footnotetext{
* Corresponding author. Present address: Deutsches Zentrum für Luft- und Raumfahrt, Rutherfordstraße 2, 12489 Berlin, Germany. Tel.: +49 3067055587.

** Corresponding author at: Institut für Raumfahrtsysteme, Universität Stuttgart, Pfaffenwaldring 29, 70569 Stuttgart, Germany. Tel.: +49 71168562511.

E-mail addresses: katharina.otto@dlr.de (K.A. Otto), srama@irs.uni-stuttgart.de (R. Srama).
}

instruments to be tested under well defined conditions. But it also enables supporting research, such as the investigation of impact ionisation plasmas from metal targets [9] or the track of a grain in an aerogel [10].

A potential of $2 \mathrm{MV}$, produced by a Van de Graaff generator, accelerates dust particles from the source to the experiment chamber [11-13]. In the dust source, grains with masses between $10^{-19} \mathrm{~kg}$ and $10^{-14} \mathrm{~kg}$ may acquire a positive charge between $0.1 \mathrm{fC}$ and $100 \mathrm{fC}$. They are then released to the accelerating potential and reach velocities between $2 \mathrm{~km} / \mathrm{s}$ and $80 \mathrm{~km} / \mathrm{s}$ [8]. The velocity $v$ depends on the charge-to-mass ratio $q / m$ and the applied voltage $U$. Energy conservation yields

$U q=\frac{1}{2} m v^{2} \Rightarrow v=\sqrt{2 U \frac{q}{m}}$.

The grains fly through a vacuum tube that connects the accelerator with the experiment. The grains' typical distances from the centre upon impact vary between $0.5 \mathrm{~mm}$ and $5 \mathrm{~mm}$, depending on the quality of the beam focussing. Thus, locating an impact site of about one micrometre has a chance of about $10^{-6}$. Therefore monitoring the focus and dust particle position is required. It is also important to monitor and control the beam focussing and steering for an effective usage of the dust source. The accelerator experiments are often limited by the amount of available dust in the source. A non-focussed beam wastes large amounts of dust that get lost in the accelerator 


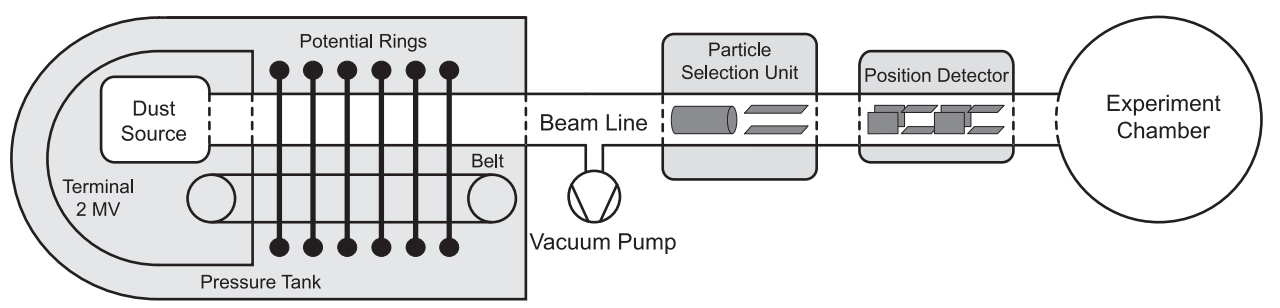

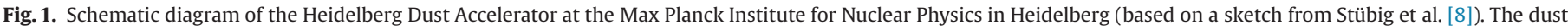

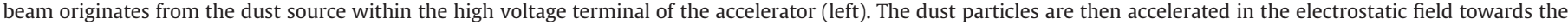

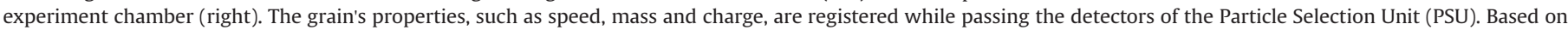
this information individual grains can be selected. The new position detector is placed behind the PSU.

(a)

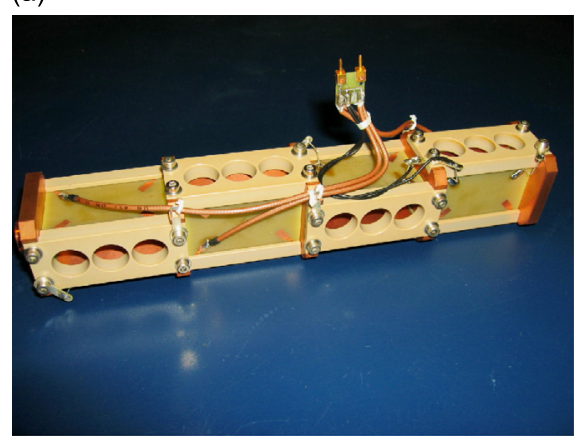

(b)

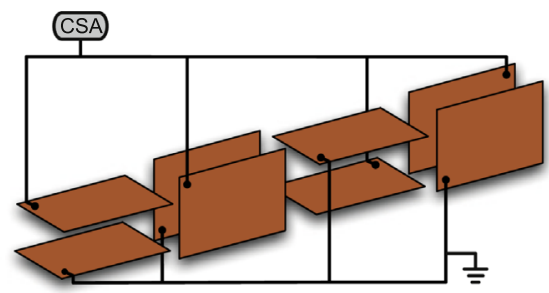

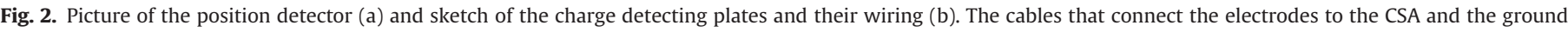

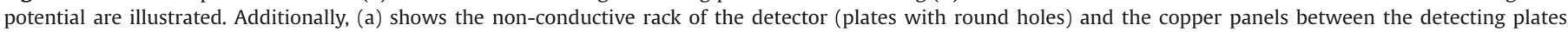
The detector has a total length of $190 \mathrm{~mm}$. The charge detecting plates have a size of $26 \mathrm{~mm} \times 38.75 \mathrm{~mm}$.

assembly. A good focus and beam alignment not only saves time by minimising the changes of the dust source (1-2 days per dust source refill), but also improves the experiment by producing more impacts on a desired area in a given time. Thus, making sure that most dust particles are focussed on an area of interest saves economic resources such as time, material and energy.

A position sensitive detector, which measures grain trajectories in a dust accelerator, has first been described by Shelton et al. [6]. The detector uses the principle of charge induction to determine the position. Depending on the distance of a charged dust particle to a conducting plate a different amount of charge is induced. This induced charge is used to infer the grains' position. The dust accelerator at the Max Planck Institute for Nuclear Physics in Heidelberg has been equipped with a new enhanced position sensitive detector which is described in this work.

This detector has been calibrated recently so that the detector signals can be mapped to a grain position. The accuracy for various signal to noise ratios (SNRs) has been determined and represents the position sensitive detector as a valuable and reliable component of the dust accelerator [14].

Other trajectory detector designs using a set of wires for charge detection have been developed and tested [15-19]. They are designed for dust trajectory and speed measurements in space. They show similar spatial accuracy, however, their signals are more complex to evaluate and often need calibration of multiple charge sensitive amplifiers (CSA). The simplicity of the signal of the newly designed detector enables the user to correlate the dust particle signal with the particle position. The user is able to easily analyse whether or not dust particles are centred and can adjust the focussing and steering of the dust accelerator, if necessary. In the future, statistics of detector signals will be used directly to adjust the accelerator properties and to focus the particles of interest automatically.

\section{Position detector design}

The new position detector includes four pairs of parallel plate electrodes made of copper printed circuit boards (PCB). The $26 \mathrm{~mm}$ $\times 38.75 \mathrm{~mm}$ plates of a pair are separated by $15 \mathrm{~mm}$. Each pair is perpendicular to the neighbouring pair. One plate of each pair is grounded, the other one is connected to a single common CSA. For the horizontal plate pairs the upper plate in one pair and the lower plate in the other pair are connected to the CSA. Similarly for the vertical plate pairs, it is the right plate in one pair and the left plate in the other. The wiring is sketched in Fig. 2(b).

The pairs are shielded from each other by grounded copper panels. The panels are $5 \mathrm{~mm}$ thick and have a central $10 \mathrm{~mm}$ round opening for the dust particles to pass through. To avoid electrical connection, the panels and plate pairs are separated by a $1.25 \mathrm{~mm}$ gap, resulting in a total detector length of $190 \mathrm{~mm}$. The plates and panels are held together by a rack of polyether ether ketone (PEEK); a non-conductive organic polymer that is compatible with vacuum conditions. A picture of the detector is shown in Fig. 2(a).

The entire detector is surrounded by a grounded $218 \mathrm{~mm}$ long and $72 \mathrm{~mm}$ wide copper cylinder such that it is inside a Faraday cage that keeps external electric fields from affecting the signals. The ends of the cylinder are shielded by conductive meshes. The CSA is attached on top of the cylinder. An external battery power supply prevents interferences such as the ones from the alternating voltage of common power supplies. This set-up is mounted inside an aluminium housing for attachment to the beam line of the dust accelerator.

A signal produced by a dust particle traversing the detector shows four charge amplitudes in which the first and third give information about the vertical and the second and fourth about the horizontal grain position. For example, a dust particle passing the detector through the centre induces four peaks with the same 


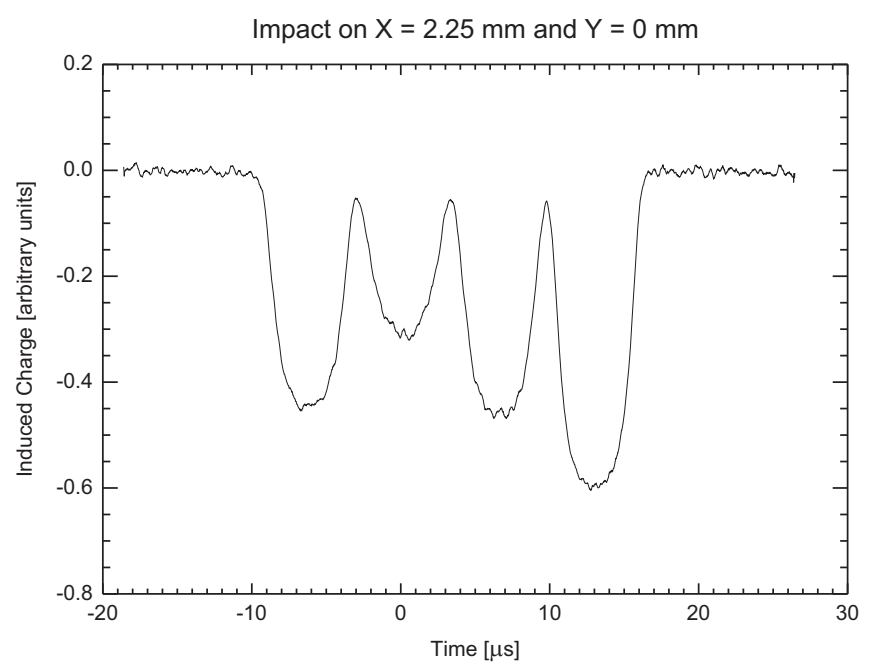

Fig. 3. A sample detector signal of a dust particle with a displacement of $(2.25$ $\pm 0.29) \mathrm{mm}$ in horizontal direction from the centre of the detector. The first and third peaks $\left(A_{1}, A_{3}\right)$, and the second and fourth peaks $\left(A_{2}, A_{4}\right)$ are associated with the detector plates for vertical and horizontal position measurements, respectively.

amplitude. A dust particle that is off to the right produces a signal as shown in Fig. 3. The first and third amplitudes are approximately equal indicating a centred position in the vertical scale. The second amplitude is smaller than the fourth amplitude representing a horizontal deflection to the right.

The set-up enables the detection of the position of a dust particle with one amplifying device only. The dust grain's charge is not needed to calculate the position. The position can be determined by knowing only the amplitude ratios and these ratios remain constant for different amounts of charge. Also, systematic gain calibration errors of the CSA are equal on each amplitude and cancel out when using an amplitude ratio.

The rise time of the amplifier is $80 \mathrm{~ns}$ which is neglectable compared to the signals time scale of roughly $30 \mu$ s (see Fig. 3). However, the capacitance of the plates can cause a delay in the decay of the amplitudes. This does not affect the amount of induced charge because the four detecting plates are electrically connected. Even though the signal does not return to the baseline between the amplitudes, the total induced charge will not exceed the image charge of the dust grain on the plate. Thus, the delay does not affect the amplitudes.

\section{Calibration}

\subsection{Set-up}

A hypervelocity dust particle impacting on a target produces impact ionisation [20-22]. Using this principle, a target plate was designed to determine the dust particle's position independently from the position detector.

The target plate has twenty-one conductive square segments of $1 \mathrm{~mm}$ side length separated by a distance of $1.25 \mathrm{~mm}$. The segments are arranged in a chess pattern. This enables five different positions each, for horizontal and vertical displacement. The segments at the corners were not used as they are lying outside the area of the circular beam line $(10 \mathrm{~mm}$ diameter). Fig. 4 shows the technical drawing of the target plate.

CSAs were attached to the rear of the target and connected to the metal-coated segments. A clear charge signal due to impact ionisation was detected when a dust particle impacted on one of the segments. An impact on the non-coated area produced a weak signal.

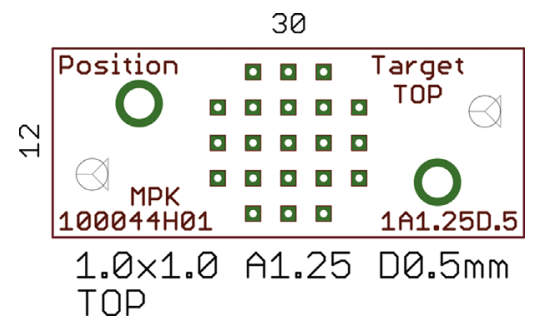

Fig. 4. Technical drawing of the target plate $(12 \mathrm{~mm} \times 30 \mathrm{~mm})$. It illustrates twenty-one squared impact segments. The segments have a side length of $1 \mathrm{~mm}$ and are separated by a distance of $1.25 \mathrm{~mm}$. The round openings ( $0.5 \mathrm{~mm}$ diameter) inside the segments were necessary for connecting the CSAs and were soldered up to provide an impact area of $1 \mathrm{~mm}^{2}$ per segment.

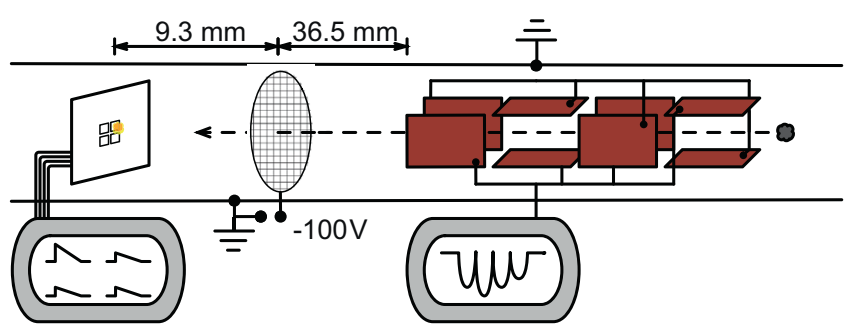

Fig. 5. Sketch of the calibration assembly. The detector is shown to the right and the segmented target to the left of the sketch. A grid with a potential of negative $100 \mathrm{~V}$ compared to the segmented target is placed between the detector and the impact target. For clarity only four segments are shown. The actual set-up was equipped with a total of twenty-one segments.

To get a more definite impact signal a grid of $70 \%$ transmission was placed between the detector and the target. A negative bias of $100 \mathrm{~V}$ was applied to the grid while the target segments were at ground potential. So the positive ions that are produced by the impact of the dust grain were drawn away from the target. The negative ions and electrons were collected by the target segments and amplified by the CSAs.

The dust accelerator was used at a voltage of $1.8 \mathrm{MV}$ to accelerate polypyrrole (PPy) coated olivine dust to about $3 \mathrm{~km} / \mathrm{s}$. Fig. 5 shows a sketch of the calibration assembly applying an impact target with only four segments for clarity.

\subsection{Results}

The position sensitive detector signals that corresponded to a segment impact were evaluated by determining the four signal amplitudes $A_{1}, A_{2}, A_{3}, A_{4}$ and calculating the ratios $A_{1} / A_{3}$ and $A_{2} / A_{4}$. A straight line was fitted to the data just before and after the detector signal. The amplitudes were determined with respect to this baseline. Four horizontal lines were fitted manually to the centre region of each amplitude where the maximum charge induction is expected.

For a future automatic analysis of the detector signals, a software programme written in Interactive Data Language (IDL) [24] working with the Canny edge detection algorithm [25] is in development. The algorithm detects the eight flanks of the signal and determines the amplitudes by averaging the data between the flanks.

Fig. 6 shows the calibration curves for $x$ - and $y$-positions. A total of 143 dust particle signals were used for this calibration. Eleven of the twenty-one available segments were impacted on. This resulted in the absence of data at $4 \mathrm{~mm}$ and $-4 \mathrm{~mm}$ in the horizontal and vertical calibration curves, respectively. For each hit target segment, the amplitude ratios were averaged and plotted against their horizontal and vertical positions. There are multiple segments with varying $x$-positions that correspond to the same $y$-position and vice versa. Thus, multiple points were plotted at each $x$ - and $y$-position. Because the impacts were uniformly distributed over each segment $\left(1 \mathrm{~mm}^{2}\right)$, a positional error of $1 \mathrm{~mm} / \sqrt{12}=0.29 \mathrm{~mm}$ was assumed. 
(a)

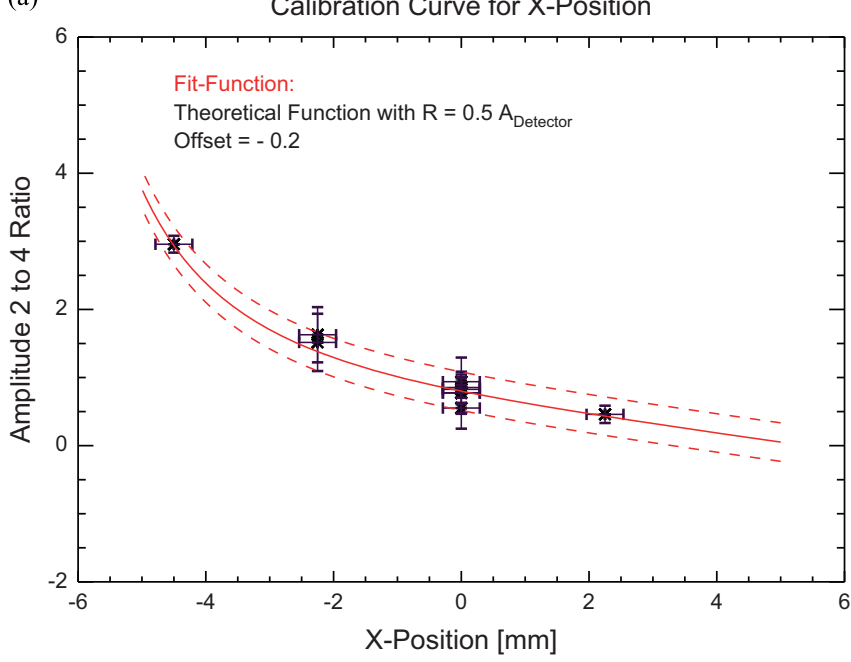

(b)

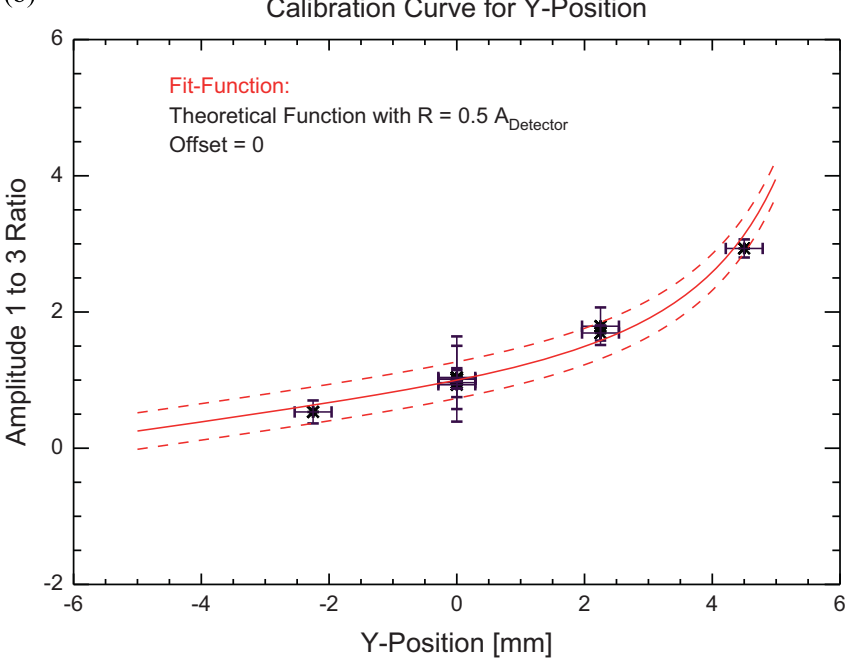

Fig. 6. Calibration curve for horizontal (a) and vertical positions (b). The plots show the correlation between the amplitude ratio and the position of a dust particle. The calibration is based on 143 detector signals. The fit was adjusted by varying the apparent detector plate size using the principle of induction between two conductive plates [23]. The dashed lines symbolise the error curves.

The standard deviation of the amplitude ratios was used for the amplitude ratio error.

The measured ratios were fitted by a function based on the principle of induction between two finite plates [23]. A charged dust grain induces an image charge in each of the opposing plates. The image charge induced in one plate induces another image charge in the opposite plate. This image charge again induces an image charge in the original plate and so on. Thus, a series of image charges $q_{n}^{\text {induced }}$ is induced. The total amount of induced charge $q^{\text {induced }}$ on each plate is given by the sum of all image charges induced on the plate:

$q^{\text {induced }}=\sum_{n=1}^{\infty} q_{n}^{\text {induced }}=\sum_{n=1}^{\infty}-q_{n-1}^{\text {induced }}\left(1-\frac{z_{n}}{\sqrt{R^{2}+z_{n}^{2}}}\right)$

Here, $z_{n}$ is the fictive distance of the $n$th image charge to the plate and $R$ is the plate size. $q_{0}^{\text {induced }}$ represents the charge of the dust particle. The induction on the panels and shielding that are not connected to the CSA were taken into account by decreasing the apparent plate size $R$.
The variations in the amplitude ratios of each segment result in an error of the calibration curves. This error was determined by averaging the ratio errors of the data points and by subtracting and adding this value from the calibration curve. The error curves are added as dashed lines in the plots of Fig. 6 and are given by \pm 0.28 for the horizontal calibration curve and \pm 0.27 for the vertical calibration curve.

In an idealised detector, a grain passing exactly through the centre would induce the same charge on both detector plates and thus produce an amplitude ratio of 1 . The calibration curve for the vertical displacement matches this criteria. However, the calibration curve for the horizontal displacement has an offset of -0.2 . This might be due to inaccuracies in the construction or installation in the beam line. Nevertheless, the agreement of both calibration curves' slopes indicates a high symmetry in the detector set-up.

\section{Discussion}

\subsection{Precision}

The determination of the detector amplitudes is limited by the noise on the detector signal. Thus, the detector shows different accuracies for different signal to noise ratios (SNRs). The SNR is defined as

$\mathrm{SNR}=\frac{A}{\sigma_{\text {signal }}}$

where $A$ is the amplitudes' sum $\left(A=A_{1}+A_{3}\right.$ and $A=A_{2}+A_{4}$ for vertical and horizontal displacements, respectively) which is the representative for the grain charge and $\sigma_{\text {signal }}$ is the standard deviation of the baseline signal in the absence of a dust grain. For high SNRs the detector is more accurate as the amplitudes can be determined more precisely. In general the lowest amplitude should not be smaller than three times the standard deviation of the baseline signal to guarantee an acceptable identification of the amplitude. Assuming that the noise is given by a Gaussian distribution, this ensures that $99.7 \%$ of the data points of an amplitude are above the baseline and thus clearly distinguishable from it. The detection limit also depends on the position of the dust particle in the detector as a grain with a large offset from the centre induces both, a high and a low amplitude. However, the amplitude sum is nearly independent of the dust particle position. Simulations have shown that it varies by about $5 \%$.

The calibration curves in Fig. 6 were used to interpret the position accuracy of the detector. The following considerations derive from the vertical calibration curve but are equally valid for the horizontal displacement, since they are both described by the same function that only differs in a fixed offset.

The error $\Delta A$ of the amplitudes $A_{1}$ and $A_{3}$ is defined as $\Delta A=3 \cdot \sigma_{\text {signal }}$ to ensure that the amplitudes are clearly distinguishable from the baseline. This results in an error in the ratio $A_{1} / A_{3}$. It is given by Gaussian error propagation:

$\Delta\left(\frac{A_{1}}{A_{3}}\right)=\frac{A_{1}}{A_{3}} \sqrt{\left(\frac{\Delta A}{A_{1}}\right)^{2}+\left(\frac{\Delta A}{A_{3}}\right)^{2}}$

It is useful to express this in terms of the amplitudes' sum $A=A_{1}+A_{3}$ and the amplitude ratio $R_{a}=A_{1} / A_{3}$. Substituting the relation between the SNR and $\Delta A(\mathrm{SNR}=3 A / \Delta A)$ yields

$\Delta R_{a}\left(R_{a}, \mathrm{SNR}\right)=\frac{3 \cdot\left(R_{a}+1\right)}{\mathrm{SNR}} \cdot \sqrt{1+R_{a}^{2}}$.

For evaluating the effect of the SNR on the accuracy of the detector, the spatial equivalent of the error of the amplitude ratio was plotted against the dust particle position for various SNRs (Fig. 7(a)). The error 
(a)

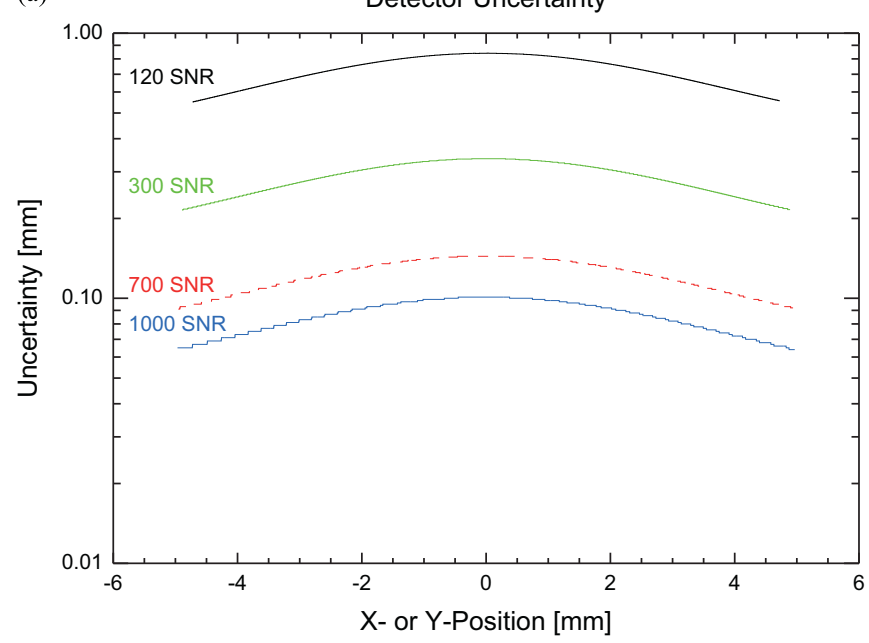

(b)

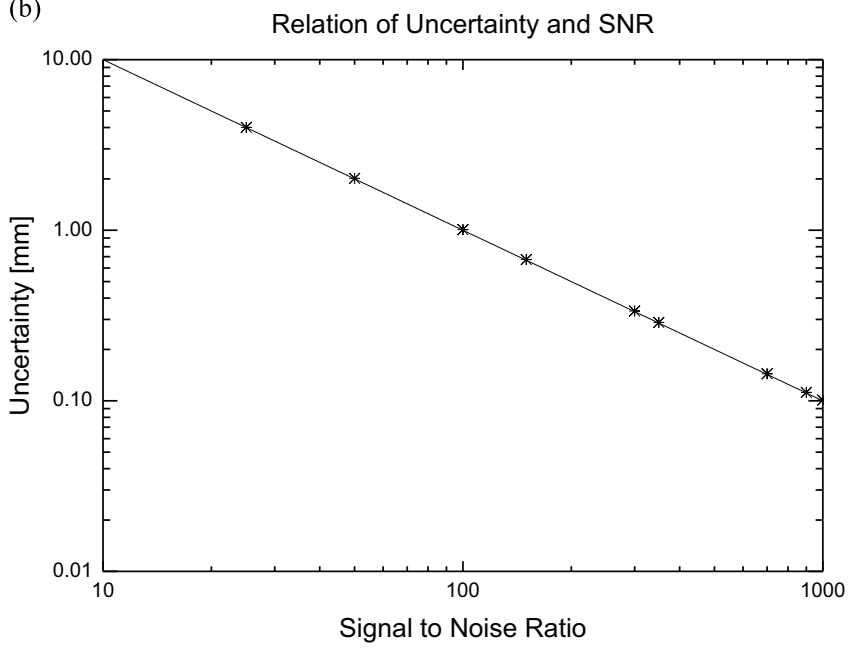

Fig. 7. Detector uncertainty as a function of the displacement from the centre for different SNRs (a). The dashed line represents a common detector SNR of 700. The steps in the curves are due to approximations made in the calculations. Note the logarithmic scale. Maximum detector uncertainty as a function of the SNR (b). The values were taken from (a) and complemented with further data points. A fit was added to approximate the missing data. Note the double logarithmic scale.

of the amplitude ratio $\Delta R_{a}$ was converted to a position error using the calibration curves (Fig. 6).

The detector shows a maximal inaccuracy when the dust particle passes through the centre (Fig. 7(a)). This can be understood because the ratio is most sensitive to noise when two amplitudes are nearly equal. The symmetry in the curves represents the symmetry of the detector.

Fig. 7(b) shows the maximum position uncertainty as a function of the SNR. The values were read off Fig. 7(a) and complemented with further data points. A fit was added so that it is possible to determine the minimum SNR necessary for a certain accuracy. The position uncertainty exceeds the detector opening of $10 \mathrm{~mm}$ for SNRs less than 10.

The typical noise level on the detector signal is given by $0.15 \mathrm{fC}$. With a representative grain charge of $100 \mathrm{fC}$ this results in a SNR of approximately 700 and thus to a detector accuracy of $0.09 \mathrm{~mm}$ to $0.14 \mathrm{~mm}$ depending on the grain position. For grains passing through the centre, the maximum error of $0.14 \mathrm{~mm}$ has to be assumed. With the beam size dimension exceeding the spatial resolution of the detector by roughly 70 times, the detector is suitable for the analysis of the steering and focussing of the accelerator.
Additionally, the detector's response to charge was evaluated. A reference charge detector [27] was used to determine the dust particle's charge. The fraction of the particle charge that is measured by the position sensitive detector is given by the amplitude sum $A$. Considering only dust grains that travelled through the centre, the data indicate that $(85 \pm 3) \%$ of the charge carried by the dust grains are induced on the detector's plates.

The detection limit of a plate is given by three times the standard deviation of the baseline signal leading to $3 \times 0.15 \mathrm{fC}=0.45 \mathrm{fC}$. For a grain passing through the centre of the detector, two equal amplitudes are produced. Thus, the grain induces $2 \times 0.45 \mathrm{fC}=0.90 \mathrm{fC}$. Because only $85 \%$ of the grain charge is detected on the plates the minimum charge $q_{c e n}^{\min }$ a dust particle has to carry in order to be noticed at the centre is $q_{c e n}^{\min }=0.90 / 0.85 \mathrm{fC}=1.06 \mathrm{fC} \approx 1.1 \mathrm{fC}$.

According to the calibration curves in Fig. 6, the maximum amplitude ratio is given by approximately 4 . Hence, for the smallest detectable signal (the smaller amplitude is three times larger than $\left.\sigma_{\text {signal }}\right)$, the amplitudes add up to $(1+4) \times 0.45 \mathrm{fC}=2.25 \mathrm{fC}$. This means that the minimum grain charge for off-centred grains $q_{o f f}^{\min }$ has to be $q_{\text {off }}^{\min }=2.25 / 0.85 \mathrm{fC}=2.65 \mathrm{fC} \approx 2.7 \mathrm{fC}$. The position of grains with charges above this value can be measured confidently with the position detector.

\subsection{Systematic uncertainties}

Effects that cause systematic uncertainties in the detector signal include the trajectory deflection due to gravity and image charges induced on the detector plates. The divergence of the dust beam is also able to manipulate the detector signal. However, most of these uncertainties have minor impacts on the detector accuracy as the following considerations demonstrate.

The deflection of the dust particle in the detector due to the gravitational acceleration of $g=9.8 \mathrm{~m} / \mathrm{s}^{2}$ can be estimated using the signal in Fig. 3. The particle roughly takes $t=25 \mu \mathrm{s}$ to traverse the detector. This causes a deflection $d_{g}$ of

$d_{g}=\frac{1}{2} g t^{2}=\frac{1}{2} \times 9.8 \frac{\mathrm{m}}{\mathrm{s}^{2}} \times\left(25 \times 10^{-6} \mathrm{~s}\right)^{2}=3.1 \mathrm{~nm}$

which is below the spatial resolution of the detector and thus can be neglected.

A traversing dust particle induces image charges on the opposing plates in the detector. These image charges exert a force $F_{C}$ on the dust grain following Coulomb's law:

$F_{C}=\frac{1}{4 \pi \epsilon_{0}} \frac{q_{\text {grain }} q_{\text {image }}}{r^{2}}$

where $1 / 4 \pi \epsilon_{0}=9.0 \times 10^{9} \mathrm{Nm}^{2} / C^{2}$ is the electric constant, $q_{\text {grain }}$ is the charge of the dust grain, $q_{\text {image }}$ is the induced image charge on the detector plate and $r$ is the distance between the fictive image charge and the dust grain (twice the distance of the dust grain to the conductive plate).

A grain that passes through the centre induces two equal charges on each plate and thus is not affected by the induced electric field. The largest deflection is given for grains with the maximum offset from the centre. A representative dust grain with a charge of $100 \mathrm{fC}$ and the largest measurable offset of $4 \mathrm{~mm}$ induces in first order $80 \mathrm{fC}$ on the closer plate $(r=2 \times 3.5 \mathrm{~mm})$ and $20 \mathrm{fC}$ on the further plate $\left(r=2 \times 11.5 \mathrm{~mm}\right.$ ) (see Fig. 6). The deflection $d_{C}$ is given by

$d_{C}=\frac{1}{2} a_{C} t^{2}$

where $a_{C}$ is the acceleration due to the Coulomb force and $t$ the traversing time of the dust particle. Using Eq. (1), $t$ is given by

$t=\frac{2 L}{v}=\frac{2 L}{\sqrt{2 U \frac{q_{\text {grain }}}{m}}}$ 
where $L$ is the length of the plates. Because the dust particles pass two plate pairs which exert a Coulomb force in the same direction, the effective length is given by $2 L$. $U$ and $m$ represent the accelerator voltage and grain mass, respectively. The Coulomb acceleration can be determined using Eq. (6):

$a_{C}=\frac{F_{C}}{m}=\frac{1}{4 \pi \epsilon_{0}} \frac{q_{\text {grain }} q_{\text {image }}}{r^{2} m}$

Substituting $t$ and $a_{C}$ in Eq. (7), $d_{C}$ is given by

$d_{C}=\frac{1}{2} \frac{1}{4 \pi \epsilon_{0}} \frac{q_{\text {grain }} q_{\text {image }}}{r^{2} m}\left(\frac{2 L}{\sqrt{2 U \frac{q_{\text {grain }}}{m}}}\right)^{2}=\frac{1}{4 \pi \epsilon_{0}} \frac{q_{\text {image }} L^{2}}{r^{2} U}$.

With an image charge $q_{\text {image }}=80 \mathrm{fC}$, a plate length $L=38.75 \mathrm{~mm}$, a distance between the grain and image charge $r=7.0 \mathrm{~mm}$ and an accelerator voltage $U=1.8 \mathrm{MV}$, Eq. (10) yields

$d_{C}=9.0 \times 10^{9} \frac{\mathrm{Nm}^{2}}{\mathrm{C}^{2}} \frac{80 \times 10^{-15} \mathrm{C} \times\left(39 \times 10^{-3} \mathrm{~m}\right)^{2}}{\left(7.0 \times 10^{-3} \mathrm{~m}\right)^{2} \times 1.8 \times 10^{6} \mathrm{~V}}$

$=1.2 \times 10^{-8} \mathrm{~m}=12 \mathrm{~nm}$.

Here, the image charge in the opposing plate (20 fC), which reduces the effective acceleration and therefore the deflection, has not been considered. However, the deflection caused by only one image charge is already small enough to show that the effect of the Coulomb force on the grain trajectories can be neglected.

A dust particle trajectory does not necessarily have to be parallel to the electrode plates due to the divergence of the beam caused by imperfect focussing of the dust accelerator. The dust trajectory begins inside the dust source where a needle with a few micrometres at the tip charges the grains [8]. The position detector has a $10 \mathrm{~mm}$ round opening ( $5 \mathrm{~mm}$ radius) and is placed approximately $5 \mathrm{~m}$ behind the dust source. Therefore, a dust particle needs to have an angle $\alpha$ of

$\tan \alpha=0.005 \mathrm{~m} / 5 \mathrm{~m}=10^{-3} \mathrm{rad}=1 \mathrm{mrad}$

or less to be able to pass the detector without colliding with the walls. For a small angle $\tan \alpha \approx \alpha$ can be assumed. Thus, the maximum divergence angle of a dust particle is approximately represented by $1 \mathrm{mrad}$. For the detector with a length of $190 \mathrm{~mm}$ this leads to a maximum displacement $d_{\alpha}$ of

$d_{\alpha}=\tan \alpha \times 0.19 \mathrm{~m}=0.19 \mathrm{~mm}$.

The effect of a common detector SNR of 700 results in a similar uncertainty $(0.14 \mathrm{~mm})$. The current detector set-up does not allow to correct for the beam divergence, however $0.19 \mathrm{~mm}$ is the maximum displacement and can be reduced by the accelerator focussing. We assumed a perfect accelerator focussing with parallel dust trajectories to calibrate the detector.

\subsection{Computer simulations}

In addition to the experimental set-up a simulation of the position sensitive detector's performance was obtained with the software package Соицомв [26]. It allows the user to calculate the induced charge in assemblies such as the position sensitive detector. The simulation was used to calculate an idealised calibration curve and detection limit.

In addition to the detector plates, the shielding panels and the inner shielding cylinder (diameter of $70 \mathrm{~mm}$ ) were considered for the simulation. For the dust particle a point charge was assumed.

To generate a calibration curve, signals with four amplitudes were generated. For each millimetre along the trajectory through the detector, the induced charge on the four detecting plates was determined. Fig. 8(a) shows the charge signal along a trajectory of $2.25 \mathrm{~mm}$ horizontal displacement. The origin of the coordinate system was placed in the centre of the detector.
The signals of twenty-three trajectories were simulated and the amplitudes of each signal $\left(A_{1}, A_{2}, A_{3}, A_{4}\right)$ were determined. The ratios $A_{1} / A_{3}$ were plotted against the $y$-position and the ratios $A_{2} / A_{4}$ were plotted against the $x$-position. As the simulation is idealised the two calibration curves are the same for the $x$ - and $y$-positions. Fig. 8(b) shows the result.

The theoretical calibration curve based on the algorithm described by Winters et al. [23] and the simulated calibration curve nearly match each other. However, the experimental calibration curve is shallower than the simulated curve. This implies that in the real detector less charge is induced on the plate that produces the larger amplitude, which might be due to the loss of induced charge to the screening and panels.

The induced charge of the simulated detector was determined and does not drop below $90 \%$. In comparison, the real detector is able to detect $(85 \pm 3) \%$ of the grain charge. Nevertheless, this demonstrates good agreement as the simulated detector neglects charge loss to further shielding that occurs in the real assembly.

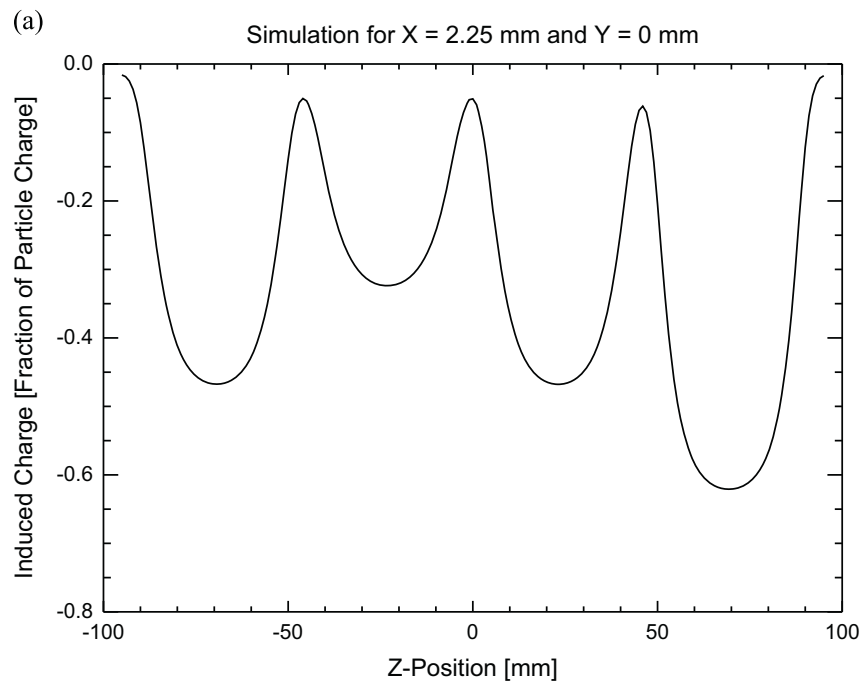

(b) Experimental, Simulated and Theoretical Calibration Curves

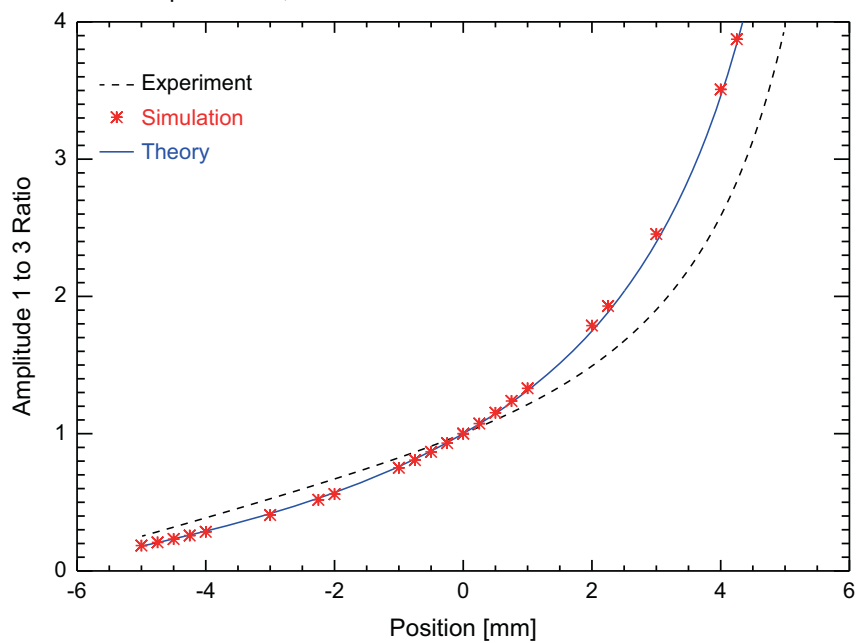

Fig. 8. A sample simulated signal that had a displacement of $2.25 \mathrm{~mm}$ in $x$-direction from the centre (a). The origin of the coordinate system is located at the centre of the detector. A simulated calibration curve was produced from the signals (b). The simulated amplitude ratios are indicated by asterisk. The experimental calibration curve (dashed line) and the theoretical expected curve for an ideal detector (solid line) are also shown. 


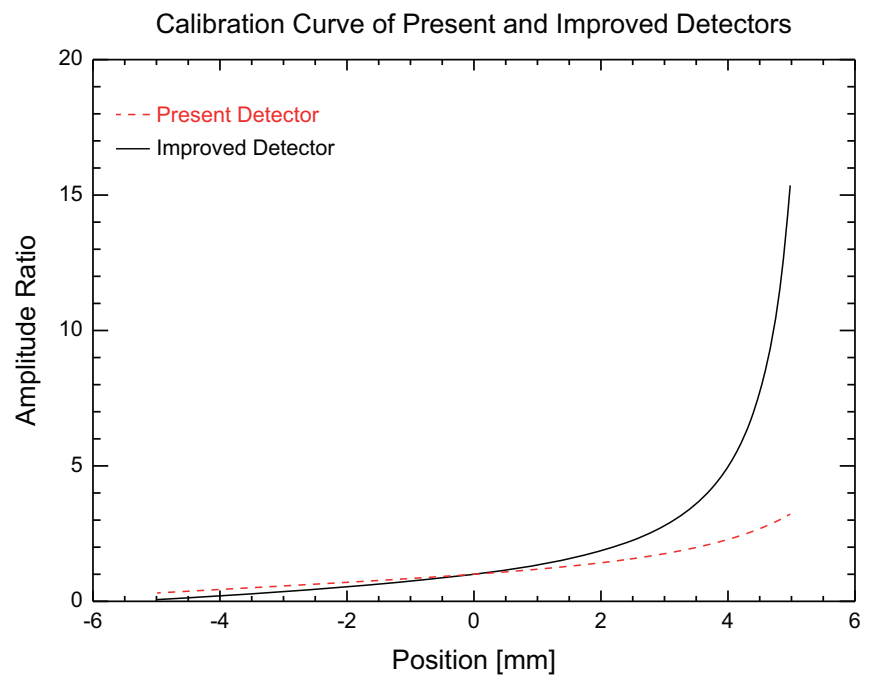

Fig. 9. Calibration curve of the present plate detector (dashed line) and theoretical improvement of the calibration curve (solid line) using a plate distance of $11 \mathrm{~mm}$.

\subsection{Suggestion for improvement}

The position detector is currently capable of measuring the position of a dust particle with an accuracy of about $0.14 \mathrm{~mm}$.

It is of interest to improve the accuracy in order to correlate impact sites and grain properties. This would greatly expand the impact studies that could be done at the accelerator. For instance, impact traces in aerogel could be correlated with a dust particle of certain properties. The same would be possible for impact crater studies on various targets. A main task to achieve this is to reduce the noise on the detector's charge amplifier and improve the detection limit.

The accuracy can also be improved by reducing the plate distance. Currently the plates are separated by $15 \mathrm{~mm}$. A reduction to $11 \mathrm{~mm}$ is possible and would in particular improve the accuracy of dust particles not passing through the centre as seen in Fig. 9. A steeper calibration curve shows less sensitivity due to noise. For centred dust particles the present accuracy of $0.14 \mathrm{~mm}$ could be improved by a factor of 1.5 resulting in an accuracy of $0.09 \mathrm{~mm}$.

\section{Conclusion}

A position sensitive detector based on charge induction was calibrated and analysed via experiments and simulations.

The detector is composed of four conductive plate pairs. In each pair, one plate is connected to a single common CSA producing a signal with four amplitudes.

A calibration set-up using a segmented impact target as an independent position measurement was implemented in the beam tube. The impact location could be ascertained for eleven different dust particle positions. The positions were mapped to the detector signals by using the ratio of two signal amplitudes. This resulted in a calibration curve for both, horizontal and vertical directions.

The experimental calibration curves were found to only differ in an offset of 0.2 but not in slope showing a high symmetry in the detector set-up.

Furthermore the accuracy of the detector was calculated and a relation between the SNR and detector accuracy was established. The calibration curve yielded an estimate of the detector accuracy of $0.09 \mathrm{~mm}$ to $0.14 \mathrm{~mm}$ depending on the dust particle position in the detector and based on a SNR of 700. The maximum error has to be assumed for dust particles passing through the centre.
About $(85 \pm 3) \%$ of the grain charge is induced on the detecting plates. With an average noise on the detector signals of approximately $0.15 \mathrm{fC}$, this leads to a general charge detection limit of about $2.7 \mathrm{fC}$. For dust particles passing through the centre of the detector, this limit is reduced to about $1.1 \mathrm{fC}$.

The assembly was simulated using the software package Coulomb [26]. The simulation was used to calculate detector signals for certain dust particle positions. They were used to produce a calibration curve that matches the theoretical calibration curve for a charge between two finite conductive plates. Additionally, the induced charge was simulated and found to not drop below $90 \%$ of the grain charge.

The experiments and simulations show good agreement. The signal shapes match well, but the fraction of induced grain charge and calibration curves differ slightly due to loss of induced charge to the shielding in the experimental set-up.

All things considered the position sensitive detector installed in the Heidelberg Dust Accelerator's beam line is a valuable and reliable device for determining a dust particle's trajectory. The detector sets the basis for future on-line adjustment of the steering and focussing of the dust accelerator.

\section{Acknowledgments}

We gratefully acknowledge the preparatory and accompanying work from G. Baust, G. Matt and A. Mocker. We would like to thank the Max Planck Institute for Nuclear Physics in Heidelberg for supporting this project.

\section{References}

[1] R. Srama, T.J. Ahrens, N. Altobelli, S. Auer, J.G. Bradley, M. Burton, V.V. Dikarev, T. Economou, H. Fechtig, M. Görlich, M. Grande, A. Graps, E. Grün, O. Havnes, S. Helfert, M. Horányi, E. Igenbergs, E.K. Jessberger, T.V. Johnson, S. Kempf A.V. Krivov, H. Krüger, A. Mocker-Ahlreep, G. Moragas-Klostermeyer, P. Lamy, M. Landgraf, D. Linkert, G. Linkert, F. Lura, J.A.M. McDonnell, D. Möhlmann, G. E. Morfill, M. Müller, M. Roy, G. Schäfer, G. Schlotzhauer, G.H. Schwehm, F. Spahn, M. Stübig, J. Svestka, V. Tschernjawski, A.J. Tuzzolino, R. Wäsch, Space Science Reviews 114 (2004) 465.

[2] E. Grün, H. Fechtig, J. Kissel, D. Linkert, D. Maas, J.A.M. McDonnell, Astronomy and Astrophysics Supplement Series 92 (1992) 411.

[3] J. Kissel, F.R. Krueger, J. Silén, B.C. Clark, Science 304 (2004) 1774.

[4] J. Kissel, ESA Special Publications 1077 (1986) 67.

[5] R. Srama, A. Srowig, M. Rachev, E. Grün, S. Kempf, G. Moragas-Klostermeyer, A. Srowig, T. Conlon, D. Harris, S. Auer, A. Glasmachers, S. Helfert, H. Linnemann, V. Tschernjawski, Earth Moon Planets 95 (2005) 211.

[6] H. Shelton, C.D. Hendricks Jr., R.F. Wuerker, Journal of Applied Physics 31 (1960) 1243.

[7] J.F. Friichtenicht, Review of Scientific Instruments 34 (1962) 209.

[8] M. Stübig, G. Schäfer, T.-M. Ho, R. Srama, E. Grün, Planetary and Space Science 49 (2001) 853.

[9] B.-K. Dalmann, Planetary and Space Science 25 (1977) 135.

[10] M.J. Burchell, S.A.J. Fairey, P. Wozniakiewicz, D.E. Brownlee, F. Hörz A.T. Kearsley, T.H. See, P. Tsou, A. Westphal, S.F. Green, J.M. Trigo-Rodríguez, G. Domingúez, Meteoritics and Planetary Science 43 (2008) 23.

[11] A. Mocker, S. Bugiel, S. Auer, G. Baust, A. Colette, K. Drake, K. Fiege, E. Grün, F. Heckmann, S. Helfert, J. Hillier, S. Kempf, G. Matt, T. Mellert, T. Munsat, K. Otto, F. Postberg, H.-P. Röser, A. Shu, Z. Sternovsky, R. Srama, Review of Scientific Instruments 82 (2011) 095111.

[12] S. Hasegawa, Y. Hamabe, A. Fujiwara, H. Yano, S. Sasaki, H. Ohashi, T. Kawamura, K.-I. Nogami, K. Kobayashi, T. Iwai, H. Shibata, International Journal of Impact Engineering 26 (2001) 299.

[13] M.J. Burchell, M.J. Cole, J.A.M. McDonnell, J.C. Zarnecki, Measurement Science and Technology 10 (1999) 41.

[14] K. Otto, Calibration of the position sensitive beam monitor of the Heidelberg Dust Accelerator, Diploma Thesis. Retrieved from MPG Publication Repository, 2011. $\langle$ http://hdl.handle.net/11858/00-001M-0000-0012-0911-6〉.

[15] S. Auer, E. Grün, S. Kempf, R. Srama, A. Srowig, Z. Sternovsky, V. Tschernjawski, Review of Scientific Instruments 79 (2008) 084501.

[16] S. Auer, Physics Chemistry, and Dynamics of Interplanetary Dust, in: B.A S. Gustafson, M.S. Hanner (Eds.), Proceedings of the 150th Colloquium of the International Astronomical Union, Gainesville, Florida, USA, 14-18 August 1995, 104, Astronomical Society of the Pacific Press, Provo, Utah, 1996, pp. 251-255. 
[17] J. Xie, Z. Sternovsky, E. Grün, S. Auer, N. Duncan, K. Drake, H. Le, M. Horányi, R. Srama, Review of Scientific Instruments 82 (2011) 105104.

[18] S. Auer, G. Lawrence, E. Grün, H. Henkel, S. Kempf, R. Srama, Z. Sternovsky, Nuclear Instruments and Methods in Physics Research Section A 622 (2010) 74.

[19] P. Northway, S. Auer, K. Drake, M. Horányi, A. Mocker, T. Munsat, A. Shu, Z. Sternovsky, E. Thomas, J. Xie, Measurement Science and Technology 23 (2012) 105902

[20] K. Hornung, Y.G. Malama, K. Thoma, Advances in Space Research 17 (1996) 77.

[21] J.F. Friichtenicht, J.C. Slattery, NASA Technical Note D-2091 (1964).

[22] H. Fechtig, E. Grün, J. Kissel, in: J.A.M. McDonnel (Ed.), Cosmic DustWileyInterscience, Chichester, Sussex, and New York, 1978, pp. 607-669.
[23] D.F.A. Winters, M. Vogel, D.M. Segal, R.C. Thompson, Journal of Physics B: Atomic, Molecular and Optical Physics 39 (2006) 3131.

[24] Exelis Visual Information Solutions, Boulder, Colorado, USA. URL 〈http://www. exelisvis.com>, 2013.

[25] J. Canny, IEEE Transactions on Pattern Analysis and Machine Intelligence 8 (1986) 679.

[26] Integrated Engineering Software, Winnipeg, Manitoba, Canada. URL 〈http:// www.integratedsoft.com>, 2011.

[27] R. Srama, S. Auer, Measurement Science and Technology 19 (2008) 055203. 